\title{
Necessity of Developing Psychological Interventions in the Management of Type II Diabetes and its Barriers: A Qualitative Study from the Perspective of Iranian Health Policymakers
}

\author{
Tahereh Ziadlou', Fahimeh Fathali Lavasani ${ }^{1}$, Adis Kraskian Mujembari ${ }^{3}$, Mohammad Reza Seirafi ${ }^{3}$, Ahmad \\ Hajebi $^{4,5 *(D)}$ \\ 1 PhD Student of Health Psychology, Department of Psychology, Karaj Branch, Islamic Azad University, Karaj, Iran \\ 2 PhD, Department of Clinical Psychology, School of Behavioral Sciences and Mental Health, Tehran Institute of Psychiatry, \\ Iran University of Medical Sciences, Tehran, Iran \\ 3 PhD, Assistant Professor of Psychology, Department of Psychology, Karaj Branch, Islamic Azad University, Karaj, Iran \\ ${ }^{4} \mathrm{MD}, \mathrm{MSc}$, Research Center for Addiction and Risky Behaviors, Department of Psychiatry, Iran University of Medical \\ Sciences, Tehran, Iran \\ 5 Professor of Psychiatry, General Director, Department of Mental Health and Substance Abuse, Iran Ministry of Health and \\ Medical Education, Tehran, Iran
}

\author{
*Corresponding author: \\ Ahmad Hajebi, Research Center for \\ Addiction and Risky Behaviors, \\ Department of Psychiatry, Iran \\ University of Medical Sciences, \\ Tehran, Iran \\ Tel: 09102122897 \\ Email: hajebi.ahmad@gmail.com
}

Received: 26 Nov. 2019

Accepted: 21 Jan. 2020

ePublished: 01 Feb. 2020

\begin{abstract}
Background and Objective: Diabetes mellitus is known as one of the health emergencies of the $21^{\text {st }}$ century. This disorder is associated with an increased risk of developing cardiovascular diseases, stroke, diabetic nephropathy, neuropathy, retinopathy, and pregnancy complications, as well as lower quality of life, high economic burden, and high risk of premature death. The aim of this study was to investigate the necessity of and barriers to developing psychological interventions to treat, improve, and manage type II diabetes from the perspective of health policymakers in Iran.

Materials and Methods: This qualitative study was conducted on 11 policymakers of the Ministry of Health and Medical Education of Iran within 2016-2019. The data were collected using semistructured and in-depth interviews and then analyzed by content analysis.

Results: The main themes related to the necessity of developing psychological interventions included impact on the quality of life, disease management, cost-effectiveness, and complications of diabetes.

Conclusions: The current study was conducted on senior health policymakers in the Ministry of Health and Medical Education of Iran. Accordingly, our findings can be used in decision making and the development, implementation, and evaluation of psychological intervention programs for type II diabetic patients in Iran.

Keywords: Diabetes mellitus, Health policy, Psychology
\end{abstract}

\section{Background}

Diabetes mellitus is known as one of the health emergencies of the $21^{\text {st }}$ century. According to the World Health Organization (WHO) statistics, this disorder has affected 422 million people around the world so far, with an almost double increase in its global incidence [1]. Diabetes is associated with an increased risk of developing cardiovascular diseases, stroke, diabetic nephropathy, neuropathy, retinopathy, and pregnancy complications. It can also lead to low quality of life, a high economic burden, and a high risk of premature death [2-4]. Diabetes as one of the major health disorders in societies is the most common disease resulting from metabolic disorders [5].

Based on the WHO statistics, the number of people with diabetes will be doubled in 2030 [6]. According to the WHO report in 2016, the prevalence rates of type II diabetes in Iran were $10.3 \%$ and $14 \%$ in the adult population and those aged $>30$ years, respectively. This disease had also the prevalence rates of $9.3 \%$ and $11.1 \%$ respectively in males and females in this country [7]. The prevalence of diabetes type II is on a rising trend worldwide. Accordingly, this disease incurs cost and burden on the healthcare system of many countries and is accompanied by mortality and morbidity given its long-term complications [8]. For example, about $2 \%, 10 \%$, and $50 \%$ of type II diabetic patients respectively get blind, develop visual impairment, and suffer from neuropathy after a diabetes 
history of 15 years. Overall, the risk of death for diabetic patients is rated twice that of non-diabetic ones [9].

Diabetic patients are required to perform most of their activities individually; accordingly, their behaviors play a key role in controlling their disease [10]. The delivery of diabetes self-management training and support is a critical element in taking care of all people with diabetes. This end involves an ongoing process of presenting the required knowledge, skills, and abilities to enable self-care, as well as the introduction of activities that help the patients to perform and maintain essential behaviors to manage their condition [11]. It is important to learn about diabetes self-management to prevent or delay its side effects. The effectiveness of diabetes self-management has been also confirmed in numerous studies suggesting such benefits as better clinical outcomes, improved quality of life, fewer cases of hospitalization, and reduced healthcare costs [12-16].

Self-management in diabetes includes healthy eating, doing physical activity, taking medications on time, following the health tips by health workers, and modifying medications and behaviors accordingly [16]. Proper control of blood glucose can prevent or delay diabetes complications, thereby not only improving patient's quality of life but also significantly reducing healthcare costs [17]. However, structured education for self-management seems unknown for diabetic patients. For instance, less than $10 \%$ of people diagnosed with type II diabetes in the UK and less than half of all diabetic patients in the United States registered for diabetes self-management education [18].

Moreover, patient adherence to self-management is at an undesirable level mainly owing to the complexity of the treatment that should be followed over a long period of time. Based on the literature, medication adherence among diabetic patients is $35.7-67.9 \%$, which was even lower for doing physical activity and following a healthy diet $[1,19]$. In the same vein, studies in Iran are indicative of poor self-management status among diabetic patients. In a study performed in Turkey [1], the lowest self-management score was reported for physical activity among patients [20].

Diabetes is a significant source of stress in life; therefore, it is not surprising that this disease is correlated with psychological disorders and comorbidities [21]. People with diabetes are twice as likely to develop depression at the clinical level [22]. In addition, $40 \%$ of diabetic patients show the symptoms of severe anxiety [23]. Diabetes also creates social pressure that can prevent social interactions as people with diabetes are often negatively judged by others [24-26]. In this regard, Schabert et al. reported that diabetic patients were concerned about unwanted attention or misinterpretation of drug abuse when using vials and syringes publicly. As a result, such stigma could prevent diabetes-related self-management in the affected patients [24]. Negative emotional states caused by stigma also prevent insulin injection in public places or delay insulin intake [25]. Another example of concealment is unwillingness to reduce the consumption of highcalorie foods for shame [26].

\section{Objectives}

Given the importance of the topic, the current study was conducted to investigate the necessity of and barriers to developing psychological interventions to treat, improve, and manage type II diabetes from the perspective of health policymakers in Iran, using qualitative content analysis.

\section{Materials and Methods}

Participants

This qualitative study was conducted using content analysis. This type of analysis is utilized to classify qualitative data in studies that aim to describe a phenomenon [27]. This study was performed after making the necessary coordination with the head of the Mental Health and Addiction Office of Ministry of Health and Medical Education in Iran and receiving an ethics code from the Ethics Committee of Islamic Azad University, Alborz Branch, Iran. The data were collected using an interview. Prior to the interview, the participants' consent was obtained to record their voice. Furthermore, they were assured about the confidentiality of their information.

The study population consisted of the policymakers of the Ministry of Health and Medical Education of Iran who were selected by the Deputy Health Minister of Health and Medical Education of Iran. The study was conducted between 2016 and 2019. The participants included internal medicine experts, epidemiologists, and social medicine experts. The study population was selected using the purposive sampling technique, and sampling was continued until data saturation (i.e. collecting new data until the repetition and stabilization of the previous data). In the present study, data saturation was obtained after the implementation of 11 interviews.

\section{Inclusion and exclusion criteria}

The inclusion criteria were work experience related to the domain of the study and willingness to participate in the study and share experiences. On 
the other hand, the exclusion criterion was dissatisfaction to continue the interviews for any reason.

\section{Data collection}

The data were collected using semi-structured and in-depth interviews. The interview began with small talk and brief explanation on the decision of Mental Health and Addiction Office of Iran Health and Medical Education Ministry to develop a protocol for psychological intervention for diabetes management in Iran according to the primary healthcare components. Each interview lasted 45-60 min. The interviews were conducted at a convenient time and place in the Medical Education building of Iran Ministry of Health.

The key questions asked included: "How do you assess the need for developing a package of psychological interventions for the treatment, improvement, and management of diabetes?", "What is the role of psychological interventions in the treatment of non-communicable diseases (e.g., diabetes), improvement of disease management, and prevention of physical and mental comorbidities?", "What do you suggest for the better implementation of psychological intervention package and better collaboration of the health team in diabetes treatment?", and "To your best knowledge and experience, what do you think are the most important barriers to the implementation and effectiveness of such interventions, and what is your suggestion for overcoming these barriers?".

In the present study, the key questions were modified or described, if necessary, according to the discretion of the interviewees. Furthermore, if needed, each interview was followed by some clarifying questions, such as "What do you mean?" or "Please explain more if you can." At the end of the interview, the participants were asked to add more complementary information, if needed. The transcripts of the interviews were prepared and analyzed precisely, and then the next interview was run. The audio files of the interviews were transcribed precisely and then analyzed by the researcher to obtain the internal and external components. Each interview was listened several times so that the researcher could find a deep understanding of the participants' conversations. Semantic units and main messages were identified after several reviews. The sections of the obtained texts were summarized and coded. In this section, each recorded word and phrase was considered as the unit of analysis.

\section{Data analysis}

In this study, raw data were classified based on the accurate interpretation and inference of the researcher. After coding, summarizing, and categorizing these concepts, the themes were extracted. A constant comparison method was used accordingly. To ensure the clarity and consistency of the data, the intercoder agreement on the coded texts with similar samples was determined, and the necessary modifications were applied [28, 29].

\section{Ethical considerations}

All related ethical considerations, including voluntary participation, oral consent, participant privacy, and confidentiality of information, were respected in this study. The present study was approved by the Ethics Committee of Islamic Azad University, Alborz Branch (IR.IAU.K.REC.1398.014).

\section{Results}

The participants in the study included 11 health policymakers at the highest level of decision-making in the Ministry of Health and Medical Education of Iran. All participants were experienced in policymaking in the field of non-communicable diseases. The participants had the age range of 45 80 years. The demographic characteristics of the study participants are presented in Table 1.

Table 2 summarizes the main themes and subthemes of the experiences of health policymakers in Iran about the necessity of and barriers to developing psychological interventions for the treatment, improvement, and management of diabetes.

In the following sections, the participants' views that were used to determine the concepts are presented.

\section{1) Impact on quality of life}

According to the study participants, one of the most important reasons for the necessity of developing a psychological intervention package for the treatment, improvement, and management of diabetes was the impact of such interventions on the quality of life among diabetic patients. The extracted sub-themes in this domain included

Table 1. Demographic variables of study participants

\begin{tabular}{lcc}
\hline Variables & Mean Frequency & SD (\%) \\
\hline Age & 53.09 & 13.5 \\
Work experience & 24 & 14.81 \\
Work experience in non-communicable diseases & 20.8 & 13.53 \\
\hline
\end{tabular}


Table 2. Main themes and sub-themes of the experiences of health policymakers in Iran about the necessity of and barriers to developing psychological interventions for the treatment, improvement, and management of diabetes

\begin{tabular}{lc}
\hline Sub-themes & Main themes \\
\hline Restriction & Impact on quality of life \\
Disease label & \\
Dependence on others & Disease management \\
Compliance with treatment & \\
Active follow-up & Cost-effectiveness \\
The importance of psychological interventions in the treatment of non-communicable diseases & Diabetes complications \\
The role of psychological interventions in treatment compliance & \\
Costs of disease control, management, and treatment & Barriers to perform interventions \\
Psychol complications of the disease & \\
Difficulty to communicate with the patient & \\
Low perceived risk in society & \\
Lack of trust in the expertise of the service provider system & \\
Patient fatigue due to long-term treatment & Need for family support \\
Lack of a referral culture in society & \\
Limited prevention services per capita &
\end{tabular}

restriction, disease labels, and dependency on others.

A) Restriction

Many participants pointed to the limitation due to illness as one of the most important factors justifying the need for developing psychological interventions to treat, improve, and manage diabetes. In this respect, one participant stated:

"Due to diabetic labeling, a patient may think that he/she has some intense limitations that severely affect his/her lifestyle. For example, be/she may think that he/she is unable to play football or do some intense physical activities or should not eat many foods, which, in turn, affect the quality of life." (An epidemiologist)

B) Disease label

Many participants pointed to disease labeling (the title of the disease), which restricts diabetes selfmanagement, as one of the reasons for the necessity of developing a related psychological intervention package. Accordingly, one participant acknowledged:

"Like other chronic diseases, diabetes has a very long-term treatment period, which may have its own consequences. No matter what happens, the label restricts the patient whether these restrictions are real or not". (An epidemiologist)

C) Dependency on others

In the present study, many participants supported the necessity of developing interventions since diabetes can lead patients to be dependent on others. According to the participants, this dependence could be due to financial need, information achievement, and medication supply. In this regard, a subject said:

"An elderly patient who does not have a good memory depends on another person for medication reminding and provision. When a person takes medicine for 30 years or more, he or she needs to visit a doctor every 3 months for medicine prescription"
2) Disease management

In the present study, many participants referred to the role of developing interventions package in disease management.

A) Compliance with treatment

One of the reasons that Iranian health policymakers supported the necessity of developing interventions was compliance with treatment. This was clearly evident in their conversations:

"A person who is educated knows how to answer to those who say, 'Eat; it is only this time.' and how to respond to those who warn him, 'Don't eat. You are a diabetic patient, this is not good for you.' He/she knows how to manage to avoid these all."

B) Active follow-up

Some participants indicated the importance of designing an intervention package in the active follow-up of the disease. In this regard, it was stated:

"A patient should be always aware of his/her blood pressure and careful of his/her blood sugar." (An expert in social medicine)

In addition, an epidemiologist pointed out:

"Attempt should be made to encourage people to change their treatment or lifestyle on their own."

C) Importance of psychological interventions in the treatment of non-communicable diseases

A great number of participants in the study emphasized the importance of psychological interventions package in the treatment of noncommunicable diseases. Regarding this, an expert in social medicine said:

"People with mental disorders, both mild and severe, who at the same time suffer from non-communicable diseases most likely do not have their illnesses under control ... those who are not aware of their mental problems mostly deny their physical illnesses."

This issue was also noted by a physician who 
argued:

"Depression caused by chronic illness as a known condition ... anxiety, and anxiety about the disease ... non-adherence to the treatment ... all these are areas where counseling is needed, anyway in psychology and psychiatry."

Additionally, a social medicine expert in this regard stated:

"People with depression disorder have much or little amount of foods in their diet. This increases or decreases glucose tolerance and affects their blood sugar... So, it is accepted that you have to control mental disorders in people with noncommunicable diseases. "

D) Role of psychological interventions in treatment compliance

Most of the health policymakers in the present study believed that psychological interventions play an important role in controlling and improving diabetes. Some of the participants' comments in this regard are as follows:

"A patient must be able to identify and remove the barriers to self-care. That means he/she must have problem-solving skills and be able to adapt and cope with the conditions."

"In any case, our healthcare system must consider the physical, psychological, and social aspects altogether, rather than just paying attention to psychological issues."

\section{3) Cost-effectiveness}

Many participants believed that designing psychological interventions could be helpful in the cost-effectiveness of diabetes treatment and control. For example, one of the participants mentioned:

"A master thesis written by an epidemiology student was about a very significant relationship between social capital and hemoglobin $A 1 \mathrm{c}$ and suggested that the higher the social capital, the lower the A1C hemoglobin levels."

\section{4) Complications of diabetes}

Another reason necessitating intervention development from the participants' perspective was the complications of diabetes. These complications were categorized into two types of physical and psychological.

A) Physical complications of diabetes

With regard to the physical complications of diabetes, one of the participants said:

"Diabetes is one of the diseases that, if left unchecked, causes serious complications, one of the most important of which is 'diabetic foot ulcer', which can even lead to death or amputation ... Diabetes complications are important and finally occur. Another complication of diabetes is related to the eyes in the form of vision loss. Diabetes can also cause kidney problems or need for dialysis and cardiovascular problems since it affects all organs."

B) Psychological complications of diabetes Considering the psychological complications of diabetes, a subject stated:
"The challenges a patient exposed to make bim/her stressful."

\section{5) Barriers to implementation of interventions}

The data obtained from interviews resulted in the identification of the barriers to implementing interventions. A total of seven sub-themes were obtained that included difficulty in patient communication, low perceived risk in the community, lack of trust in the expertise of the service provider, patient fatigue due to exposure to long-term treatment, lack of family support, lack of referral culture in society, and limited per capita prevention services.

A) Difficulty in communicating with the patient

An expert in social medicine in this regard pointed out:

"Cultural differences are among the major barriers to communication."

Furthermore, another participant stated,

"Our practitioners used to be successful because they knew the culture of the place, they would know how to deal with people. They might be tanght some skills in their curriculum though I am not sure, but it's important that staff know how to talk to people since they have to deal with people. However, unfortunately, in Iran, doctors do not listen to and communicate with their patients."

Additionally, an epidemiologist referred to this issue as follows:

"Specialists often speak in media which is not effective because they speak a language that is not understandable by the audience."

B) Low perceived risk in society

Low perceived risk among patients was cited by many participants as a barrier to developing interventions. For instance, an expert in social medicine contended:

"In Iran, people mostly believe that they do not develop any complications; it happens to others; it does not happen to me." Similarly, an epidemiologist clearly asserted:

"Riske perception is low in Iran."

In addition, another expert in social medicine said: "We warn diabetic patients if they do not control their sugar blood level, they shall suffer from kidney problems in 5 years, their vision shall decrease, and they may face retina bleeding. Besides, they may experience kidney failure in 20 years and lose their vision in 25 years. But they ignore the long-term consequences."

B) Lack of trust in the expertise of service providers People's lack of trust in the expertise of service providers was raised as another reason urging the need for the development of intervention packages. This lack of trust was clearly indicated by a social medicine expert as he said:

"People believe that if something is cheap, it is worthless. This problem has been reported by family doctors since the 
early years of healthcare plan; they mentioned that as visits to doctors are free, people think that the doctors are not expert and experienced, so they look for other doctors."

C) Patient fatigue due to long-term treatment According to the policymakers, diabetic patients get tired of prolonged treatment and daily physical monitoring. Therefore, this issue could be taken as another reason for the necessity of intervention development.

D) Need for family support

The need for family support was another reason indicated by the participants. This was evidently stated by a subject:

"We shall change the eating habits of a family rather than an individual, which means all the family should develop bealthier eating habits instead of having a member on diet."

E) Lack of referral culture in society

Some participants also referred to the lack of referral culture in our society as another reason necessitating the development of intervention packages for diabetic patients. In this regard, a subject stated:

"One major drawback to our culture, which actually is not a cultural problem, is that people want to be provided with the highest level of service; this is our biggest challenge in the field. See, all the statistics show that we can control diabetes at the primary health care level, but what are we doing? All caregivers, all of those who come to us, want to visit diabetes ad endocrinology specialists to control their problems"

G) Limited per capita prevention services

The presence of limited prevention services per capita was also indicated as a reason for the importance of developing interventions. Accordingly, one of the participants argued:

"One of the major challenges is the expenses. There is a limited budget for the delivery of prevention services per capita. If you do not consider the budget and start to expand services, then you have already accepted to reduce the quality of services."

\section{Discussion}

Based on the findings, the main themes related to the necessity of developing psychological interventions included impact on the quality of life, disease management, cost-effectiveness, and complications of diabetes. Many studies reported the role of diabetes in reducing the quality of life in patients [30-33]. Quality of life in diabetic patients is so important that some studies have suggested that the early diagnosis and treatment of diabetes improve life quality [30]. The diabetes guidelines clearly focus on the importance of achieving and maintaining the quality of life that is considered a major goal in the healthcare system [31].

Several studies also highlighted the importance of psychological interventions in the improvement of the quality of life in patients with chronic diseases. For example, Fernandez et al. investigated the influence of problem-solving skills on patients' quality of life and self-esteem and found that problem-solving skills significantly improved the dimensions of quality of life in patients, including their cognitive function, health perception, mental well-being, and physical function [32]. In another study, Wattana et al. showed that self-management programs promoted the quality of life among Thai patients with type II diabetes [33]. The findings of the present study also indicated the necessity of developing psychological interventions to improve the quality of life among diabetic patients.

From the perspectives of Iranian health policymakers, the development of psychological interventions is required due to their influence on the management of type II diabetes. Consistent with our findings, Noordali et al. introduced learning coping techniques and self-care as important steps in managing diabetes [21]. Hampson et al. also showed that psychological interventions can lead to better treatment compliance and therapeutic outcomes among diabetic patients [34]. Furthermore, the results of other studies are indicative of the positive effects of motivational interviewing on hemoglobin A1c (HbA1c) level reduction, fear of low blood sugar levels, and improved quality of life [35-37]. In this regard, in a systematic review and meta-analysis, Sherifaliet al. showed that the implementation of psychological interventions among diabetic patients led to a slight improvement in HbA1c levels and suggested to apply these techniques, along with therapeutic approaches, in diabetic patients [35].

In another study, Harvey concluded that psychological interventions were influential in adherence to everyday care for diabetes [36]. Given the importance of psychological interventions in the management and improvement of diabetes, Christie et al. studied children and adolescents with type I diabetes and found that receiving these interventions did not lead to a decrease in HbA1c among children with poor control. However, they considered such interventions essential for the management of diabetes disease [38]. According to similar studies, it seems that the provision of psychological interventions for diabetic patients may result in useful findings, which was clearly stated by health policymakers in Iran.

Another implication of the present study was the cost-effectiveness of psychological interventions in the management and treatment of diabetes. Many studies reported the importance of psychological interventions in reducing the expenses related to 
chronic diseases, such as diabetes. For example, in a systematic review, Hampson et al. showed that psychological interventions could shorten the length of hospital stay in diabetic patients and therefore, reduce treatment costs and improve disease management [34]. Riegel et al. also suggested the effectiveness of the motivational interviewing method in reducing hospital readmission in patients with heart failure [39]. Consistent with the previous studies, the findings of the present study demonstrated the necessity of psychological interventions to reduce costs associated with the improvement and control of diabetes.

The policymakers also suggested the complications of diabetes as another reason urging the need for developing psychological interventions to improve diabetes management and treatment. Given the high number of diabetes complications [2-4], it is necessary to develop psychological interventions to improve and control this disease and its associated complications.

Finally, factors, such as difficulty in communicating with patients, low perceived risk in society, lack of trust in the expertise of service providers, patient fatigue due to prolonged treatment, need for family support, lack of referral culture in society, and limited per capita prevention services, were introduced as the most important barriers to providing psychological interventions among diabetic patients by the participants in this study. Similar to our research, Chapman et al. conducted a qualitative study on 23 physicians in China to identify the most important barriers to providing psychological services to type II diabetic patients. In the mentioned study, the major barriers to the interventions included physicians' knowledge and skills, time constraints, and lack of financial incentives, as well as other barriers, such as social perception (mental health treatment is less important than physical health), neglect of policymakers, deliberate disregard for psychological care by physicians, and doubts about the effectiveness of psychological care [40].

In another qualitative study, Blixen et al. showed that interventions to optimize communication between patients and service providers could provide useful findings for eliminating the barriers to self-management among diabetics [41]. In their systematic review, Sohal et al. suggested that diabetes programs that focus on the improvement of communication, consideration of prevailing misconceptions, and adoption of culture-specific strategies could improve diabetes management among patients in South Asia [42].

One of the prominent strength of this study is its qualitative design since the findings can provide a deep understanding of the necessity of and barriers to developing psychological interventions for the management of type II diabetes that could not be achieved through quantitative studies. However, the findings reported in this study have certain limitations. Firstly, voluntary participation made room for the exclusion of the experiences of those who did not wish to participate in the study for any reason. In addition, the data were collected only from health policymakers, and type II diabetes patients were not included in the study. Regarding this, complementary studies are recommended to be performed on type II diabetes patients.

\section{Conclusions}

The present study was conducted on senior health policymakers in the Ministry of Health and Medical Education of Iran. Therefore, our findings can be used in decision making and the development, implementation, and evaluation of psychological intervention programs for type II diabetic patients in Iran. Given the necessity of developing psychological interventions in the treatment, improvement, and management of type II diabetes and considering the barriers to the implementation of such interventions, it seems essential to consider such programs in the primary healthcare system of the country.

\section{Acknowledgments}

This article is part of a Ph.D. thesis in Health Psychology submitted to Islamic Azad University, Karaj Branch. The authors of this study appreciate the contributors. This research was funded by the Centers of Non-Communicable Disease Control and Mental Health Department, Social and Addiction Office of the Islamic Republic of Iran Ministry of Health and Medical Education.

\section{References}

1. Dilekler I, Doğulu C, Bozo Ö. A test of theory of planned behavior in type II diabetes adherence: The leading role of perceived behavioral control. Current Psychology. 2019; 7:1-10. [DOI:10.1007/s12144-019-00309-7]

2. Ausili D, Rossi E, Rebora P, Luciani M, Tonoli L, Ballerini E, et al. Socio-demographic and clinical determinants of selfcare in adults with type 2 diabetes: a multicentre observational study. Acta Diabetologica. 2018; 55(7):691702. [DOI:10.1007/s00592-018-1135-x] [PMID]

3. Scollan-Koliopoulos M, Bleich D, Rapp KJ, Wong $\mathrm{P}$, Hofmann CJ, Raghuwanshi M. Health-related quality of life, disease severity, and anticipated trajectory of diabetes. The Diabetes Educator. 2013; 39(1):83-91. [DOI:10.1177/ 0145721712467697] [PMID]

4. Bommer C, Heesemann E, Sagalova V, Manne-Goehler J, Atun R, Bärnighausen $\mathrm{T}$, et al. The global economic burden of diabetes in adults aged 20-79 years: a cost-of-illness study. The Lancet Diabetes \& Endocrinology. 2017; 5(6):423-30. [DOI:10.1016/S2213-8587(17)30097-9] [PMID]

5. Shaw JE, Sicree RA, Zimmet PZ. Global estimates of the prevalence of diabetes for 2010 and 2030. Diabetes Research and Clinical Practice. 2010; 87(1):4-14. [DOI:10.1016/j.diabres.2009.10.007] [PMID]

6. Rathmann W, Giani G. Global prevalence of diabetes: 
estimates for the year 2000 and projections for 2030. Diabetes Care. 2004; 27(10):2568-9. [DOI:10.2337/diacare. 27.10.2568] [PMID]

7. Lotfi MH, Saadati H, Afzali M. Prevalence of diabetes in people aged $\geq 30$ years: the results of screening program of Yazd Province, Iran, in 2012. Journal of Research in Health Sciences. 2014; 14(1):87-91. [PMID]

8. Pal K, Dack C, Ross J, Michie S, May C, Stevenson F, et al. Digital health interventions for adults with type 2 diabetes: qualitative study of patient perspectives on diabetes selfmanagement education and support. Journal of Medical Internet Research. 2018; 20(2):e40. [DOI:10.2196/jmir. 8439] [PMID] [PMCID]

9. Huang ES, Laiteerapong N, Liu JY, John PM, Moffet HH, Karter AJ. Rates of complications and mortality in older patients with diabetes mellitus: the diabetes and aging study. JAMA Internal Medicine. 2014; 174(2):251-8. [DOI: 10.1001/jamainternmed.2013.12956] [PMID] [PMCID]

10. Chatterjee S, Davies MJ, Heller S, Speight J, Snoek FJ, Khunti K. Diabetes structured self-management education programmes: a narrative review and current innovations. The Lancet Diabetes \& Endocrinology. 2018; 6(2):130-42. [DOI:10.1016/S2213-8587(17)30239-5] [PMID]

11. Beck J, Greenwood DA, Blanton L, Bollinger ST, Butcher MK, Condon JE, et al. 2017 National standards for diabetes self-management education and support. The Diabetes Educator. 2017; 43(5):449-64. [DOI:10.1177/01457217 17722968] [PMID]

12. Strawbridge LM, Lloyd JT, Meadow A, Riley GF, Howell BL. One-year outcomes of diabetes self-management training among medicare beneficiaries newly diagnosed with diabetes. Medical Care. 2017; 55(4):391-7. [DOI:10.1097/ MLR.0000000000000653] [PMID]

13. He X, Li J, Wang B, Yao Q, Li L, Song R, et al. Diabetes selfmanagement education reduces risk of all-cause mortality in type 2 diabetes patients: a systematic review and metaanalysis. Endocrine. 2016; 55(3):712-31. [DOI:10.1007/ s12020-016-1168-2] [PMID] [PMCID]

14. Chrvala CA, Sherr D, Lipman RD. Diabetes selfmanagement education for adults with type 2 diabetes mellitus: a systematic review of the effect on glycemic control. Patient Education and Counseling. 2016; 99(6):92643. [DOI:10.1016/j.pec.2015.11.003] [PMID]

15. Pillay J, Armstrong MJ, Butalia S, Donovan LE, Sigal RJ, Chordiya $\mathrm{P}$, et al. Behavioral programs for type 1 diabetes mellitus: a systematic review and meta-analysis. Annals of Internal Medicine. 2015; 163(11):836-847. [DOI:10.7326/ M15-1399] [PMID]

16. Veazie S, Winchell K, Gilbert J, Paynter R, Ivlev I, Eden KB, et al. Rapid evidence review of mobile applications for selfmanagement of diabetes. Journal of General Internal Medicine. 2018; 33(7):1167-76. [DOI:10.1007/s11606-0184410-1] [PMID] [PMCID]

17. Dobson R, Whittaker R, Jiang $Y$, Maddison R, Shepherd M, McNamara $C$, et al. Effectiveness of text message based, diabetes self management support programme (SMS4BG): two arm, parallel randomised controlled trial. BMJ. 2018; 361:k1959. [DOI: 10.1136/bmi.k1959] [PMID] [PMCID]

18. Powers MA, Bardsley I, Cypress M, Duker P, Funnell MM, Fischl AH, et al. Diabetes self-management education and support in type 2 diabetes: a joint position statement of the American Diabetes Association, the American Association of Diabetes Educators, and the Academy of Nutrition and Dietetics. Clinical Diabetes. 2016; 34(2):70-80. [DOI:10.2337/diaclin.34.2.70] [PMID] [PMCID]

19. Polonsky WH, Henry RR. Poor medication adherence in type 2 diabetes: recognizing the scope of the problem and its key contributors. Patient Preference and Adherence. 2016; 10:1299-307. [DOI:10.2147/PPA.S106821] [PMID] [PMCID]

20. Ansar-Zadeh S, Saeid A, Mahmoodi Z, Salehi L. Determinants of diabetes self-management in women with gestational diabetes with PRCEED model approach.
Iranian Journal of Endocrinology and Metabolism. 2019; 20(5):212-23.

21. Noordali F, Cumming J, Thompson JL. Effectiveness of mindfulness-based interventions on physiological and psychological complications in adults with diabetes: a systematic review. Journal of Health Psychology. 2017; 22(8):965-83. [DOI:10.1177/1359105315620293] [PMID]

22. Anderson RJ, Freedland KE, Clouse RE, Lustman PJ. The prevalence of comorbid depression in adults with diabetes: a meta-analysis. Diabetes Care. 2001; 24(6):1069-78. [DOI:10.2337/diacare.24.6.1069] [PMID]

23. Grigsby AB, Anderson RJ, Freedland KE, Clouse RE, Lustman PJ. Prevalence of anxiety in adults with diabetes: a systematic review. Journal of Psychosomatic Research. 2002; 53(6):1053-60. [DOI:10.1016/s0022-3999(02)004178] [PMID]

24. Schabert J, Browne JL, Mosely K, Speight J. Social stigma in diabetes: a framework to understand a growing problem for an increasing epidemic. The Patient. 2013; 6(1):1-10. [DOI:10.1007/s40271-012-0001-0] [PMID]

25. Tak-Ying Shiu A, Kwan JJ, Wong RY. Social stigma as a barrier to diabetes self-management: implications for multi-level interventions. Journal of Clinical Nursing. 2003; 12(1):149-50. [DOI:10.1046/j.1365-2702.2003.00735.x] [PMID]

26. Wellard SJ, Rennie S, King R. Perceptions of people with type 2 diabetes about self-management and the efficacy of community based services. Contemporary Nurse. 2008; 29(2):218-26. [DOI:10.5172/conu.673.29.2.218] [PMID]

27. Mayring P. Qualitative content analysis. A Companion to Qualitative Research. 2004; 1:159-76.

28. Boeije H. A purposeful approach to the constant comparative method in the analysis of qualitative interviews. Quality and Quantity. 2002; 36(4):391-409. [DOI:10.1023/A:1020909529486]

29. Coghlan A, Filo K. Using constant comparison method and qualitative data to understand participants' experiences at the nexus of tourism, sport and charity events. Tourism Management. 2013; 35:122-31. [DOI:10.1016/j.tourman. 2012.06.007]

30. Trikkalinou A, Papazafiropoulou AK, Melidonis A. Type 2 diabetes and quality of life. World Journal of Diabetes. 2017; 8(4):120-9. [DOI:10.4239/wjd.v8.i4.120] [PMID] [PMCID]

31. Adriaanse MC, Drewes HW, van der Heide I, Struijs JN Baan CA. The impact of comorbid chronic conditions on quality of life in type 2 diabetes patients. Quality of Life Research. 2016; 25(1):175-82. [DOI:10.1007/s11136-0151061-0] [PMID] [PMCID]

32. Fernandez O, Baumstarck-Barraun K, Simeoni MC, Auquier P. Patient characteristics and determinants of quality of life in an international population with multiple sclerosis: Assessment using the Musi Quality of life and SF-36 questionnairs with psoriasis. Multiple Sclerosis Journal. 2011; 17(10):1238-49. [DOI:10.1177/1352458511407951] [PMID]

33. Wattana C, Srisuphan W, Pothiban L, Upchurch SL. Effects of a diabetes self-management program on glycemic control, coronary heart disease risk, and quality of life among Thai patients with type 2 diabetes. Nursing \& Health Sciences. 2007; 9(2):135-41. [DOI: 10.1111/j.1442-2018. 2007.00315.x] [PMID]

34. Hampson SE, Skinner TC, Hart J, Storey L, Gage H, Foxcroft $D$, et al. Effects of educational and psychosocial interventions for adolescents with diabetes mellitus: a systematic review. Health Technology Assessment. 2001; 5(10):1-79. [DOI:10.3310/hta5100] [PMID]

35. Sherifali D, Bai JW, Kenny M, Warren R, Ali MU. Diabetes self-management programmes in older adults: a systematic review and meta-analysis. Diabetic Medicine. 2015; 32(11):1404-14. [DOI:10.1111/dme.12780]

36. Harvey JN. Psychosocial interventions for the diabetic patient. Diabetes, Metabolic Syndrome and Obesity. 2015; 
8:29-43. [DOI:10.2147/DMSO.S44352] [PMID] [PMCID]

37. Christie D, Channon S. The potential for motivational interviewing to improve outcomes in the management of diabetes and obesity in paediatric and adult populations: a clinical review. Diabetes, Obesity \& Metabolism. 2014; 16(5):381-7. [DOI:10.1111/dom.12195] [PMID] [PMCID]

38. Christie D, Thompson R, Sawtell M, Allen E, Cairns J, Smith $\mathrm{F}$, et al. Effectiveness of a structured educational intervention using psychological delivery methods in children and adolescents with poorly controlled type 1 diabetes: a cluster-randomized controlled trial of the CASCADE intervention. BMJ Open Diabetes Research and Care. 2016; 4(1):e000165. [DOI:10.1136/bmjdrc-2015000165] [PMID] [PMCID]

39. Riegel B, Masterson Creber R, Hill J, Chittams J, Hoke L. Effectiveness of motivational interviewing in decreasing hospital readmission in adults with heart failure and multimorbidity. Clinical Nursing Research. 2016; 25(4):36277. [DOI:10.1177/1054773815623252] [PMID] [PMCID]

40. Chapman A, Yang H, Thomas SA, Searle K, Browning C. Barriers and enablers to the delivery of psychological care in the management of patients with type 2 diabetes mellitus in China: a qualitative study using the theoretical domains framework. BMC Health Services Research. 2016; 16:106. [DOI:10.1186/s12913-016-1358-x] [PMID] [PMCID]

41. Blixen CE, Kanuch S, Perzynski AT, Thomas C, Dawson NV, Sajatovic M. Barriers to self-management of serious mental illness and diabetes. American Journal of Health Behavior. 2016; 40(2):194-204. [DOI:10.5993/AJHB.40.2.4] [PMID] [PMCID]

42. Sohal T, Sohal P, King-Shier KM, Khan NA. Barriers and facilitators for type-2 diabetes management in South Asians: a systematic review. PloS One. 2015; 10(9):e0136202. [DOI:10.1371/journal.pone.0136202] [PMID] [PMCID] 